\title{
Tahar Djaout, romancier du verbe libre
}

\section{Malika Kebbas}

\section{OpenEdition}

\section{Journals}

Édition électronique

URL : http://journals.openedition.org/recherchestravaux/404

DOI : $10.4000 /$ recherchestravaux.404

ISSN : 1969-6434

Éditeur

UGA Éditions/Université Grenoble Alpes

\section{Édition imprimée}

Date de publication : 30 juillet 2010

Pagination : 47-54

ISBN : 978-2-84310-174-8

ISSN : 0151-1874

\section{Référence électronique}

Malika Kebbas, «Tahar Djaout, romancier du verbe libre », Recherches \& Travaux [En ligne], 76 | 2010, mis en ligne le 30 janvier 2012, consulté le 08 septembre 2020. URL : http://journals.openedition.org/ recherchestravaux/404; DOI : https://doi.org/10.4000/recherchestravaux.404 


\section{Tahar Djaout, romancier du verbe libre}

Tahar Djaout, écrivain algérien francophone, est né à Oulkhou (petit village de la Kabylie maritime) en janvier 1954. Il obtient une licence de mathématiques à l'université d'Alger puis un DEA en sciences de l'information et de la communication à l'université Paris II. En 1975, il débute sa carrière de journaliste à l'hebdomadaire Actualités de l'émigration, puis il collabore à la rubrique culturelle du quotidien El Moudjahid qu'il quitte pour l'hebdomadaire Algérie-Actualités, dont il dirige la rubrique culturelle. En 1992, avec des amis journalistes, dont Arezki Metref et Abdelkrim Djaad, il fonde l'hebdomadaire indépendant Ruptures.

Parallèlement à son métier de journaliste, Tahar Djaout mène une carrière littéraire, en premier lieu en tant que poète. En 1972, à l'âge de dix-huit ans, il publie son premier poème dans la revue bruxelloise Le Journal des poètes. Suivront de nombreuses publications dans d'autres revues, entre autres Action poétique, Le Fou parle, Poésie. Il est également l'auteur de plusieurs recueils de poésies: Solstice barbelé (1975), L’Arche à vau l'eau (1978), Insulaire et Cie (1980), L'Oiseau minéral (1982).

À partir de 198I, il se consacre également au roman. Il nous laisse une œuvre remarquable de lucidité et de sensibilité : L'Exproprié (198I), Les Chercheurs d'os (1984), L'Invention du désert (1987), Les Vigiles (I99I), Le Dernier Été de la raison (publié à titre posthume en 1999). Dans ses romans, Tahar Djaout démonte les mécanismes d'un monde en déliquescence, qu'il tente de reconstruire différemment. Ses mots en liberté dessinent un monde en accord avec son rêve de fraternité, de paix et de générosité. C'est ce que nous nous proposons d'analyser dans cet article. 


\section{Écrire pour «différer la fêlure»}

Paraphrasant le poète palestinien Samih El Kacem, Tahar Djaout écrit :

Le silence c'est la mort

Et toi, si tu parles, tu meurs

Si tu te tais, tu meurs

Alors, parle et meurs.

Son verbe militant et engagé exprimait, à travers ses écrits poétiques, romanesques et journalistiques, le rêve d'une Algérie républicaine. Poète, Tahar Djaout l'était plus que tout, déclarant au journaliste Mohamed ZianeKhodj, moins d'une année avant son lâche assassinat : "Dans la poésie il y a le désir de déconstruire le monde et de le reconstruire différemment ${ }^{\mathrm{I}}$.»

Déconstruire le monde par le verbe poétique, tel est encore le projet qui l'anime dans ses cinq romans. Déconstruire le monde pour mettre à nu le système qui sévit dans l'Algérie nouvellement indépendante. Dire, quel qu'en soit le prix, pour abattre le mur contre lequel butent les Algériens. Il écrit en exergue de L'Exproprié :

Écrire toujours par intérim ? C'était surtout avec cela que je voulais en finir. Pour moi, il s'agissait de tenir l'équilibre assez longtemps de parler en mots en tacts en vibrations pour différer la fêlure et les picotements d'abeilles.

L'écrit journalistique ne lui suffisait plus pour «différer la fêlure», il lui fallait la liberté de la poésie et du roman, pour dénoncer le sort fait à l'Algérien, coincé entre l'État policier et l'État islamiste, passant sans autre alternative d'un dogme à l'autre.

\section{L'ère des vigiles}

Dans ses romans, Tahar Djaout décrit un État policier qui se maintient grâce à la délation et qu'incarne Skander Brik, appariteur à la mairie, ancien moudjahid (combattant de la guerre d'indépendance) :

Skander Brik fait partie de la police informelle [...] il est chargé de lui rapporter tous les faits et gestes de quelque importance dont la ville est le théâtre. Ce travail discret mais soutenu, il l'effectue avec une constante diligence, et sa curiosité, sous des dehors très réservés, est toujours en éveil. Skander Brik a fait sienne une ancienne stratégie : il est tel un insecte aux antennes ultra-sensibles qui se barricade dans sa carapace, mais conserve ses sens en éveil comme autant de pièges posés sur le chemin des imprudents².

I. Le Jeune Indépendant, août 1992.

2. Les Vigiles, Seuil, I99I, p. 49 (désormais V). 
La comparaison avec un insecte traduit le côté sournois du personnage : sous des dehors insignifiants, il n'en est pas moins dangereux, car il a la patience de «l'araignée» ( $V$, p. I68) qui tisse sa toile dans l'ombre et en silence afin de mieux y emprisonner ses proies. Cette pratique ignoble touche tous les rouages de la société, même l'école :

[...] ils [les enseignants] les [les élèves] amènent même à dénoncer les parents qui consomment de l'alcool. [...]. Mahfoudh ose espérer que Redhouane n'en est pas encore à la délation. $(V$, p. 66)

C'est le règne d'un État policier où toute expression libre est exclue et qui ne connaît pour règle que la censure :

Il y vient des journalistes (travaillant dans le quotidien Le Militant incorrigible ou l'hebdomadaire Le Vigile) qui y déversent les imprécations et y développent les analyses qu'ils ne peuvent pas imprimer, des cinéastes qui y racontent les films qu'il leur est interdit de tourner, des écrivains qui y parlent des livres qu'ils auraient écrits s'ils avaient eu la moindre chance d'être publiés. ( $V$, p. 29)

En effet, tous ceux qui menacent le pouvoir sont marginalisés, comme le déclare Mahfoudh Lemdjad lors de la cérémonie organisée par les autorités locales pour l'honorer :

Vous savez certainement que les gens qui font dans les choses dites du savoir ou de l'esprit sont rarement sollicités pour parler. Alors, ils ont comme subi une atrophie de la langue. ( $V$, p. 193)

L'État policier est maintenu par un groupe d'individus qui se réclament d'une légitimité révolutionnaire et en profitent pour s'accaparer les biens. Ainsi, ils habitent tous des "villas» qu'ils se sont appropriées lors de l'Indépendance. Nulle beauté dans leur univers; les fleurs qui ornaient leurs jardins ont été arrachées :

[...] les fleurs avaient vite disparu, remplacées par des carrés de salades, d'oignons et de tomates [...] ( $V$, p. 169)

La même idée est déjà présente dans Les Chercheurs d'os'

Ma villa est entourée d'une bonne surface cultivable. Mais les étrangers sont des frivoles. Des fleurs et des plantes odoriférantes, voici ce qu'ils ont eu l'idée de planter dans une si bonne terre. J'ai déjà commencé l'arrachage et je vois d'ici les oignons, carottes et navets qu'une terre si généreuse pourra me dispenser en automne. (CO, p. 26)

La frénésie de s'accaparer des biens touche l'ensemble de la population :

[...] mais les consommateurs parlent aussi du temps présent, de la manière d'avoir des biens et des postes dans l'administration. [...] Il y a donc tant de gens heureux sur terre qui parlent de camions, de magasins, de bâtiments [...] (CO, p. I2I)

3. Les Chercheurs d'os, Seuil, coll. «Points», 1984 (désormais CO). 
Les «vigiles» cultivent l'«illusion des mots» dans des discours et des cérémonies qui sont des "mascarades». Les discours officiels sont des morceaux d'anthologie en matière de langue de bois. Ce qu'illustre si bien le discours des chefs militaires dans Les Chercheurs d'os...

[...] d'interminables discours sur la patrie et la fraternité, de gigantesques flambeaux allumés un peu partout pour signifier le règne retrouvé de la lumière, une générosité sans balises qui faisaient du bien de chacun le bien de tout le monde. ( $C O$, p. Io)

[...] malgré la vigilance d'un chef militaire de l'armée libératrice qui portait un casque colonial et faisait à longueur de journée des discours sur le profane et le sacré, sur le courage et la couardise, sur le licite et l'interdit. (CO, p. I2)

... et le discours du maire dans Les Vigiles:

Au nom de Dieu clément et miséricordieux. Nous sommes rassemblés ce soir comme les membres d'une famille unie afin de célébrer une victoire précieuse ajoutée au palmarès chaque jour plus long des victoires naissantes. [...] L'intérêt que nos gouvernants portent à la science, la considération qu'ils témoignent aux hommes de savoir sont signifiés aujourd'hui par la présence à nos côtés de M. l'officier supérieur du commandement régional, $M$. le sous-préfet et d'autres personnalités prestigieuses que le manque de temps ne me permet pas de nommer. Ces hommes, qui ont mené la guerre libératrice, suivent de près aujourd'hui cette autre guerre contre l'ignorance et pour l'élévation du pays à l'échelle des nations prospères. ( $V$, p. 190-192)

Mahfoudh n'est pas dupe de ce discours ni de la cérémonie, pas plus que l'assistance d'ailleurs :

La perspective de ce moment ne l'émeut néanmoins pas outre mesure, car il sait que les gens gavés de discours répétitifs, de directives sibyllines et de recommandations irréalisables n'écoutent plus depuis longtemps ce qu'on leur raconte; ils réagissent seulement, quelle que soit la teneur du discours, de la seule manière qu'on attend d'eux : en applaudissant. ( $V$, p. 193)-

C'est l'«illusion des mots», ces mots qui glissent sur l'auditoire qui attend «avec docilité l'heure de passer aux choses sérieuses» $(V$, p. 193), «les choses tangibles» ( $V$, p. 190), qui se réduisent aux choses bassement matérielles, "préservant de la sorte la pérennité de leurs préoccupations œsophagiques» ( $V$, p. 56$)$. Sous le contrôle de ces individus, l'Algérie ne devient plus qu'un vaste estomac.

\section{Des «vigiles» aux frères vigilants}

Tahar Djaout décrit dans ses romans un système qui mène l'Algérie «droit dans le mur", à l'image de ce train, conduit par des gardiens en uniforme, qui menace de dérailler ${ }^{4}$ :

4. L'Exproprié, Alger, Enag Éditions, 2002, (désormais E). 
Et maintenant avec ce foutu soleil, le train semble à chaque fois louper les rails; il se met alors à tourner comme une noria dans un ciel balafré et sableux (E, p. 24).

Le fonctionnement de ce système favorisera la montée de l'islamisme, que Tahar Djaout décrit avec force dans son dernier roman :

Ceux-ci [les gardiens en uniforme] abattaient tous les astres sur notre parcours et appelaient avec force implorations la nuit définitive. Ils fomentaient probablement une orgie de sang; ils troquèrent leurs casquettes contre des turbans soigneusement repassés et laissèrent tous pousser la moustache. Ils se préparaient pour l'ultime violence. ( $E$, p. 24).

C’est dire si ce texte, écrit en 1981, démontre de façon prémonitoire la responsabilité de l'État policier dans la victoire de l'islamisme, qui a conduit le pays au chaos durant «la décennie noire».

L'islamisme, qui est dépeint avec lucidité dans Le Dernier Été de la raison', fable politique terrifiante, est présenté comme l'aboutissement inexorable de l'état policier dont l'avènement a fini par installer le règne de la mort et "par réduire l'autre au silence avant de l'effacer" (DER, p. I44). Aux "vigiles» de l'état policier, succèdent "les frères vigilants" de l'état islamiste, eux aussi comparés à des "araignées", ces insectes sournois qui attendent patiemment d'ingurgiter leurs proies:

Un jour, les gens se sentirent fatigués de penser, une lassitude s'abattit sur l'intelligence, et la raison vacilla. Ceux qui attendaient, araignées tisseuses d'obscurité, araignées patientes et résolues, que la pensée trébuche et abdique, sortirent alors et se répandirent. Comme la tombée irrévocable de la nuit. (DER, p. II4)

Ce dernier roman, ultime geste de résistance de l'écrivain, prend valeur de testament : ne pas laisser la raison vaciller, réveiller l'intelligence.

\section{Monde de l'enfance, monde des mots}

À une logique suicidaire, Tahar Djaout oppose, dans tous ses romans, le monde de l'enfance, celui du narrateur de L'Exproprié, celui de l'adolescent de quatorze ans des Chercheurs d'os, celui de Menouar Ziada et de Mahfoudh Lemdjad des Vigiles, et celui de Boualem Yekker du Dernier Été de la raison. Monde du rêve, de la beauté de la nature, de l'éveil aux sens, de l'amour, de la communion fraternelle, un monde où tout est encore possible. Monde de la poésie, des mots en liberté qui enflent et emportent, dans un élan vertigineux, «l'illusion des mots» et le silence définitif:

5. Le Dernier Été de la raison, Seuil, I999, (désormais DER). 
Il faut maintenir plus longtemps l'illusion. Je suis petit enfant. ( $E$, p. 48)

Les mots, torrent impétueux, coulant en rangs serrés, sans aucune ponctuation, compacts, se déversent comme un flot qu'on ne peut endiguer, comme le montre ce passage de L'Exproprié :

[...] et quand le soleil se leva sur le village aux arbres glaireux ses grives étaient déjà parties mais on pouvait toujours poser des pièges - et c'est l'essentiel - car les fauvettes et les chardonnerets commençaient à tout envahir ils venaient planer par dizaines sur les champs verts et bientôt déflagration du groupe sur les fleurs blanches des fèves ce qui avait tout d'abord surpris l'enfant c'était la ressemblance frappante de ces fleurs avec les oiseaux blanches et ailées avec une pointe de noir sur le cou exactement comme les bergeronnettes et l'enfant enfoncé dans l'herbe jusqu'aux aisselles s'emparait de la prairie. (E, p. II7-I2I)

Dans Le Dernier Été de la raison, c'est la forme poétique qui prend le relais, à la faveur d'une lettre adressée par Boualem Yekker à sa fille, dont il se remémore l'enfance :

Je rentrerai de voyage

et te trouverai endormie.

Le raffut des meubles se sera tu,

Les bêtes en douceur se seront éclipsées,

Et tous les tambours de la maison

Seront redevenus peaux vivantes mais discrètes.

J'arrive toujours dans la suspension juste

Des pulsations,

Quand la chaux, l'argile et leur blancheur

Ont tout occupé.

J'arrive

Et vois peu à peu l'émersion

Toi d'abord qui orchestres couleurs et mouvements,

Redonnes leur tapage aux bestioles,

Diriges des vols périlleux.

Puis les objets,

Fiers de leurs prouesses,

Déclenchent l'élan des manèges.

Tu chercheras les chiens acrobates

Du rêve

Entre les draps étonnés,

Tu secoueras un à un les poudroiements de lumière,

Et la vie se réinstallera

Tu te réveilles

Et la maison devient un carnaval. (DER, p. 77-78)

Des pages entières, sans ponctuation, d'une grande intensité poétique voient les mots se succéder à un rythme saccadé, attestant de la subversion 
des normes du roman. L'écriture oppose au paysage aride et au noir silence de la mort celui de la vie foisonnante et de l'enfance retrouvée. Geste de résistance qui empêche de sombrer dans le défaitisme, et qui, à ce titre, éclaire l'intelligence et la raison. Ainsi conclut l'adolescent des Chercheurs d'os dont les yeux se dessillent et les illusions se dissipent au terme de l'errance, véritable parcours initiatique :

Combien de morts, au fait, rentreront demain au pays ? Je suis certain que le plus mort d'entre nous n'est pas le squelette de mon frère qui cliquette dans le sac avec une allégresse non feinte. (CO, p. 156)

Cet adolescent se réincarne dans Les Vigiles, en Mahfoudh Lemdjad, jeune ingénieur versé dans la création et l'innovation. Le vieux métier à tisser qu'il a rénové symbolise l'esprit d'initiative et de créativité, mais il traduit aussi une manière d'être-au-monde. L'acte de tisser qui renvoie métaphoriquement au geste de l'écriture, au texte comme ouvrage et enchevêtrement de signes rêvant le monde dans sa diversité, se dresse en faisant barrage à tous "les inventeurs de désert".

Les livres qui ont su libérer l'homme, qui ont fait de Boualem Yekker, dans Le Dernier été de la raison, un observateur lucide, perdent à présent de leur puissance symbolique et de leur efficacité pédagogique. Ne lui restent pour se maintenir en vie, dans ce vaste désert que devient l'Algérie, que son passé et sa mémoire.

Parler devient ainsi un moyen pour conjurer le silence de la mort, pour inventer le monde, le reconstruire. C'est aussi opposer à la barbarie les mots généreux qui stimulent la raison et l'intelligence. Construire une Algérie républicaine, dont tous les censeurs seraient bannis, construire une Algérie de la beauté, de la liberté, de l'amour et de la fraternité, de la modernité, une Algérie à l'image de la cité idéale comme la rêve Boualem Yekker dans Le Dernier Été de la raison:

Dans la ville oppressante où il vivait et où il vit encore, Boualem Yekker avait échafaudé - oh ! il n’ose plus le faire - des rêves sur la cité idéale où il aimerait vivre et voir s'épanouir ses enfants. Il y aurait d'abord de la verdure - arbres et pelouses - beaucoup de verdure qui fournirait l'ombre, la fraîcheur, les fruits, la musique des feuilles et les gîtes d'amour. Il y aurait des créateurs de beauté, de rythmes, d'idylles, d'édifices, de machines. Mais aucun strapontin n'était prévu pour les régulateurs de la foi, les surveillants des consciences, les gardiens de la morale, les fondés de pouvoir du Ciel. Boualem Yekker aspirait à une humanité libérée de la hantise de la mort et du châtiment éternel. (DER, p. 67-68)

Parler, c'est aussi pouvoir se libérer du "Texte ligoteur» (DER, p. 59), du "Texte assené à coups de bâton" (DER, p. 63), du 
Texte qui muselle le monde par son intransigeance, sa beauté - qui ne tolère que l'acquiescement. Le Texte jaloux, tyrannique, qui n'admet aucune autre parole, aucune autre figure signifiante." (DER, p. II5)

Parler, c'est pouvoir enfin répondre "oui» à la question qui clôt son œuvre romanesque : "Le printemps reviendra-t-il ?».

Tahar Djaout, fidèle à ses pairs, et en particulier à Mouloud Mammeri pour qui il avait une grande admiration, avait le verbe haut, le verbe libre, celui de la vie foisonnante des rêves d'enfant. Un verbe qui conjure le silence de la mort programmée - "une véritable expérience de laboratoire», disait Mouloud Mammeri. Il a parlé pour anéantir la bête immonde, pour faire reculer les "thérapeutes de l'esprit», ceux qui, écrivait-il dans Le Dernier Été de la raison,

[...] ont compris le danger des mots, de tous les mots qu'ils n'arrivent pas à domestiquer et à anesthésier. Car les mots, mis bout à bout, portent le doute, le changement. Il ne faut surtout pas que les mots entretiennent l'utopie : c'est un acide qui creuse, dans l'opacité du dogme, des trous où se loge la controverse, où prolifèrent les questions. Ceux qui défiant l'injonction, s'agrippent aux mots incontrôlés, doivent être mis hors d'état de nuire. Par le bâillonnement, la liquidation si nécessaire.» (DER, p. I24)

Tahar Djaout était de «ceux qui défiant l'injonction, s'agrippent aux mots incontrôlés». Il l'aura payé de sa vie. 